\title{
Journal of HIV and AIDS
}

\section{Coreceptor Usage of Syncytium-Inducing-HIV-1 Isolates Depends on the Target Cell and Evolves during Pediatric Infection}

\section{Cintia M Crudeli ${ }^{1,2 \#}$, Guillermo Corró ${ }^{1,2^{*}}$, Carlos A Rocco', Natalia Beltramone', Silvia A Marino ${ }^{1}$ and Luisa Sen ${ }^{1,2}$}

'Laboratorio Biología Celular y Retrovirus, Hospital de Pediatría "Prof. Dr. Juan P. Garrahan”, Buenos Aires, Combate de los Pozos 1881 (C1245AAL) Buenos Aires, Argentina

${ }^{2}$ Consejo Nacional de Investigaciones Cientificas y Tecnológicas (CONICET), Buenos Aires, Combate de los Pozos 1881 (C1245AAL) Buenos Aires, Argentina

"These two authors have equally contributed to this work

"Corresponding author: Guillermo Corró, Hospital de Alta Complejidad "Cepernic-Kirchner" SAMIC, Avda Jorge Newbery 453 (9405) El Calafate, Santa Cruz, Argentina, Tel: +54 29669 729443; E-mail: gcorro2@gmail.com
Received date: 13 March, 2015; Accepted date: 27 April, 2015; Published date: 01 May, 2015.

Citation: Crudeli CM, Corró G, Rocco CA, Beltramone N, Marino SA, et al. (2015) Coreceptor Usage of Syncytium-Inducing- HIV-1 Isolates Depends on the Target Cell and Evolves during Pediatric Infection. J HIV AIDS Volume1.1: http:// dx.doi.org/10.16966/2380-5536.102

Copyright: (c) 2015 Crudeli CM et al. This is an open-access article distributed under the terms of the Creative Commons Attribution License, which permits unrestricted use, distribution, and reproduction in any medium, provided the original author and source are credited.

\begin{abstract}
Comparisons between different phenotypic and sequence-based bioniformatic methods for coreceptor usage prediction were performed to establish the role and variation of syncytium-inducing (SI) HIV-1 phenotype on CCR5 and CXCR4 coreceptor usage along the course of vertical infection.

Thirty-one SI HIV-1 isolates were studied (12 from the acute and 19 from the chronic stage). Infection capacity dependent on CXCR4 only (X4) or on both CXCR4 and CCR5 (R5X4) was determined by re-infection on two cell systems: PHA-activated PBMCs and GHOST coreceptorransfected cells using CXCR4 blockers when necessary.

Coreceptor usage strikingly differed between those two cell systems. On GHOST cells $95 \%$ of SI-isolates were dual tropic (R5X4). On PBMCs, strains obtained at acute infection were predominantly R5X4 (75\%), while $70 \%$ of SI HIV-1 from chronic stages was X4. In addition, four V3loop-sequence-based algorithms were applied to the previously mentioned isolates in order to classify them as R5 or X4/R5X4 variants (the net charge, $11 / 25$ rule, PSSM and geno2pheno). Only $66 \%$ of SI isolates were classified as X4 using the bioinformatic algorithms. Our study demonstrated that computational algorithms were notoriously inaccurate in predicting tropism of SI-isolates, particularly for those obtained at primary infection since half of them were misclassified as R5 variants by three of the V3-sequence-based phenotype-predictor algorithms. In conclusion, SI variants coreceptor usage may vary according to the method used. Tropism determination of SI strains on PBMCs may more closely indicate the viral behavior in vivo since we use the natural virus target. Differences along the course of infection, such as predominance of dual-R5X4 tropic in the acute stage and prevalence of X4 variants in chronic infection, probably reflect the evolution of coreceptor usage of S variants during pediatric infection. We also provide strong evidence suggesting that coreceptor usage of HIV-1 should be carefully determined before CCR5 blockers were implemented, particularly in pediatric infection.
\end{abstract}

Keywords: HIV-1; Coreceptors; Children

\section{Introduction}

HIV-1 CXCR4 using variants emerge in almost $50 \%$ of chronically infected patients $[1,2]$. The finding of CXCR4-using HIV-1 strains in around $20 \%$ of isolates from early stages of infection has been documented in adults as well as in children [3-5]. It was demonstrated that CXCR4using variants were more pathogenic than viruses that exclusively utilize the CCR5 coreceptor and its emergence has been associated with rapid T-cell depletion leading to disease progression [6-8]. In vitro, X4/X4R5 strains but not R5 induce syncytium formation in MT-2 cell line that expresses $\mathrm{CD}^{+}$and CXCR4 but not CCR5 receptors [9]. Therefore, HIV-1 tropism was initially classified as SI (syncytium-inducing) and NSI (non-syncytium-inducing) variants as equivalents for X4/X4R5 and R5, respectively. Dual-tropic variants may have a different preference in coreceptors usage and can be further classified as R5>X4 (CCR5 preference or dual-R) X4>R5 (CXCR4 preference or dual-X) and R5 X4 [10-14].

The need for a rapid and reliable assay for tropism determination has become crucial since the development of small-molecule inhibitors of CCR5 as antiretroviral (ARV) drugs. Currently, the biologic assay Monogram Trofile seems to have reached a sensitivity and sensibility high enough to cover the clinical needs [15]. Methods based on HIV-1 env sequences had a predictive accuracy of $71 \%$ to $91 \%$ [16-19] and are not useful to warrant a safe implementation of CCR5 blockers in the therapy.

The presence of SI/CXCR4-using viruses in the early stages of vertical transmission may be particularly relevant in pediatric immunopathogenesis, especially at birth, due to the extremely active thymus with a relative high number of potential CXCR4 (naïve) target cells in the newborn [12-14].

HIV-1 tropism is mainly determined by CCR 5 and CXCR 4 coreceptor binding, but the evolution of coreceptor usage of SI variants along time of infection remains unsolved $[20,21]$.

Based on this evidence, the aim of our study was to investigate whether SI variants differ in coreceptor usage in the acute or chronic clinical stages of pediatric infection, and to evaluate the accuracy of HIV-1 cell tropism determination of some of the most commonly used V3-loop-sequencebased bioinformatic algorithms in order to warrant a safe treatment prescription when a CCR5 blocker will be included in the ARV treatment.

\section{Methods}

\section{Studied population}

Thirty-nine HIV-1 vertically infected children who were followed up 
at the "Garrahan Pediatric Hospital" in Buenos Aires, Argentina, were included in the study. Informed consent was obtained from their parents or legal guardians in agreement with the Institutional Ethics Committee.

Children were classified according to HIV-1 phenotype and age at sampling: SI isolates were obtained from 12 infected infants in the acute/ primary stage ( $\leq 18$ months) and 19 children with chronic infection $(>18$ months), while NSI isolates obtained from 8 patients at different infection stages were used as controls.

\section{Isolation of HIV-1 from periphera- blood mononuclear-cell co- cultures}

HIV-1 was isolated using PBMC co-cultivation as previously described by the AIDS Clinical Trials Group [22]. Briefly, HIV-1 seronegative blood donors PBMCs pre-stimulated for 24 to 72 hours with phytohemaglutinin (PHA) $(5 \mu \mathrm{g} / \mathrm{mL}$ Difco Laboratories), were co-cultured with PBMCs from the patient at a final concentration of $2 \times 10^{6} \mathrm{cells} / \mathrm{ml}$. Co-cultures were weekly refed with PHA-pre-stimulated HIV-1 negative PBMCs and maintained for 28 days in RPMI 1640 (Gibco BRL, Invitrogen) medium supplemented with $20 \%$ fetal bovine serum (FBS), $5 \mathrm{U} / \mathrm{ml}$ IL-2 (Sigma Aldrich) and $10 \mu \mathrm{g} / \mathrm{ml}$ gentamicin (Gibco BRL Invitrogen). Measurement of HIV-1 p24 Ag of co-culture supernatants was performed every 3 days with a commercial assay kit (Vironostika HIV-1 Antigen, BioMérieux) and $\mathrm{p} 24+$ supernatants were stored at $-80^{\circ} \mathrm{C}$ for further studies.

\section{Determination of syncytium-inducing HIV-1 isolates on MT-2 cells}

The SI assay was performed as described by Japour et al. [9]. In 96well plates, $1000 \mathrm{pg}$ p24 (coculture supernatants) were added to $5 \times$ $10^{4}$ MT-2 cells/well in RPMI 1640 supplemented with 10\% FBS and 10 $\mu \mathrm{g} / \mathrm{ml}$ gentamicin. SI primary viral isolates and a CXCR4-tropic LAI laboratory adapted strain (NIH AIDS Reference and Reagent Program) were used as positive controls, and wells without virus addition and with NSI isolates were considered as negative controls. Each supernatant was tested in quadruplicate. Syncytium formation of MT-2 cells was evaluated by optic microscopy every 3 days for 28 days. Cells harvested from cultures without syncytia were further analyzed for HIV-1 proviral DNA using nested PCR to confirm or rule out infection, as described by Albert and Fenyo [23]. In addition, to assure infectivity of the virus inoculum, a parallel control assay was performed adding the same viral inoculum in wells containing $2 \times 10^{5}$ PHA-stimulated PBMC from HIV1 seronegative donors. Supernatants from all the wells were measured for p24 Ag. When the viral inoculum infected PBMCs but was unable to induce syncytium formation with absence of HIV-1 proviral DNA in MT-2 cells, the viral isolate was classified as NSI. Once HIV-1 tropism was determined, MT-2 supernatants containing the SI variants were stored at $-80^{\circ} \mathrm{C}$. Since CXCR4 but not CCR5 is expressed on the MT- 2 cells surface [24], only HIV-1 X4 and/or R5X4 strains can be harvested from MT-2 infected-cell supernatants. Further studies were performed to characterize and distinguish X4 from R5X4 on two cell systems, PBMCs and HIV-1 coreceptor transfected GHOST cell lines.

\section{Determination of coreceptor usage on GHOST cells}

SI HIV-1 variants co-receptor usage was further analyzed on a panel of GHOST cells expressing CD4/CCR5, CD4/CXCR4 or only CD4 receptors. Viral infection was performed as described by Vodrös and Fenyo in 2001 [25]. GHOST cell lines (provided by Dr. H. Salomon, INBIRS) were maintained in DMEM medium (Hyclone, Byodinamics) containing $10 \%$ FBS, and antibiotics. 500 TCID $_{50}$ were used to infect $1.5 \times 10^{5}$ cells. Infection was evaluated using fluorescence microscopy and supernatant p24 Ag measurement. In all cases, R5-tropic (HIV-1 ${ }_{B a L}$ ) and X4-tropic (HIV- $\left.1_{\mathrm{LAI}} / \mathrm{PBMC}\right)$ laboratory adapted strains were used as control of coreceptor usage. CXCR4 usage was confirmed by adding AMD3100
$(10 \mu \mathrm{g} / \mathrm{ml})$, a specific CXCR4 inhibitor. According to the infectivity on GHOST cells, the viral isolates were classified as: $\mathbf{R 5}$, only infecting GHOST-CCR5 cells; dual R (R5>X4) with a higher infectivity on cells expressing CCR5 than on those expressing CXCR4; R5X4 (dual R5 X4) having a similar infectivity on GHOST-CCR5 and GHOST-CXCR4 cell lines, dual X $(\mathrm{X} 4>\mathrm{R} 5)$ with a higher infectivity on cells expressing CXCR4 than on those expressing CCR5; or X4 those isolates that only infected CHOST-CXCR4 cells.

\section{Coreceptor usage of SI variants tested on peripheral blood mononuclear cells}

SI HIV-1 subclassification as X4 and R5X4 variants was also achieved by infection on PHA activated PBMCs, with or without CXCR4 blockage. A pool of PBMC from three HIV-1 negative blood donors was incubated with AMD-3100 for 2 hours at $4^{\circ} \mathrm{C}$, followed by infection with $500 \mathrm{TCID}_{50}$ of SI variants. To test the efficiency of the blocker, treated cells were also challenged with the R5 (Bal) and X4 (LAI) strains. In addition, NSI-strain isolates from primary cellular co-cultures were assayed as R5-positive controls. Seven and 14 days post-infection, p24 Ag was measured in the culture supernatant. If p24 was undetectable in the AMD-3100-treated PBMCs, the SI strain was considered as an X4 variant. On the contrary, if virus could be recovered the SI viral isolate was considered as an R5X4 variant. Each SI variant was tested in triplicate and the infectious capacity of isolates was evaluated without AMD-3100.

\section{HIV-1 genotyping by V3 sequence analysis and viral tropism determination}

RNA was extracted from $50 \mu \mathrm{L}$ of cell free virus supernatants from different cell system using the PureLink ${ }^{\mathrm{TM}}$ Viral RNA/DNA Mini Kit (Invitrogen $^{\mathrm{TM}}$, Carlsband, USA) Diagnostics Systems). cDNA obtained by RT-PCR was used as a template in a nested PCR for a fragment spanning the V3 to V5 region of the env gene as described by Delwart [26]. PCR amplicons were purified and directly sequenced using the DYEnamic ET terminator Cycle sequencing kit (Amersham Bioscience, England) with an ABI PRISM 310 automated sequencer. Alignments with subtype reference sequences from Los Alamos database (available at URL: www. hiv-web.lanl.gov) were generated using Clustal X [27] and edited with BioEdit version 5.0.9 [28] for visual inspection and manual correction.

In order to evaluate the prediction of HIV tropism, four different bioinformatic methods based on the HIV-1-V3-loop sequence were tested:

The net charge rule: A net charge of $\geq+5$ for the V 3 loop corresponds to CXCR4 coreceptor usage, whereas a net charge of $<+5$ is associated with CCR5 coreceptor usage [29].

The 11/25 rule: Sequences with positively charged amino acids at codons 11 and/or 25 of the V3 loop, were classified as CXCR4-using variants.

The position-specific scoring matrix: The PSSM uses background genetic variation as a baseline comparison to facilitate comparison of the residues of a sequence fragment and those of a group of aligned sequences known to have the desired property. PSSMX4/R5, which is the PSSM trained with X4 and R5 data, and PSSMSI/NSI, which is the PSSM trained on SI and NSI strain. Data are available at: http://indra.mullins.microbiol. washington.edu/webpssm/ [30].

The Geno2Phen: Based on a statistical learning method called a support vector machine (SVM). For this study the default false-positive rate (FPR) of 0.1 was chosen to yield a specificity of approximately $90 \%$ (http://www.geno2pheno.org). A more recent version of this algorithm was also developed to include clinical data (CD4\%, number of sequence ambiguities, host CCR5 $\Delta 32$ heterozygosity, and presence of insertions/ 
deletions) as input variables [31]. However, since this model was based on data from adult population, it is not applicable in the pediatric population, mainly because of differences in the $\mathrm{CD}^{+} \mathrm{T}$ cells counts.

\section{Statistical analysis}

For comparison of the V3 loop net charge and the values obtained by the PSSM for X4 and R5X4 variants we used the rank sum test of Wilcoxon-Mann-Whitney (StatXact 3.0). In all cases, two-tailed tests were performed with a significance level of 0.05 .

\section{Results}

\section{Phenotypic determination of coreceptor use of SI HIV-1 isolates according to the stage of pediatric infection}

To investigate whether SI HIV-1 variants may differ in coreceptor usage at different clinical stages of infection, a group of vertically infected children carrying SI HIV-1 variants was evaluated. MT-2 supernatants (SI viral stocks) were further classified as X4 or dual-tropic (R5X4) on two different cell systems. Thirty-one SI isolates were tested on PBMCs and 24 of them were also evaluated on a panel of GHOST cells as described in Methods.

Coreceptor usage on PBMCs: 31 SI variants were evaluated for coreceptor usage by infecting PBMCs in the presence or absence of the CXCR4 blocker AMD-3100. Only 14 (48\%) of them were dual-tropic while the remaining 17 isolates (52\%) exclusively used the $\mathrm{X} 4$ coreceptor (Table 1). By using this culture method, SI variants from early stages of infection seemed to be predominantly R5X4 variants while most of the SI variants isolated from chronically infected patients exclusively used the CXCR4 coreceptor $(\mathrm{p}<0.01)$ (Table 2$)$.

Coreceptor usage on transfected GHOST cell lines: 24 out of the 31 SI isolates described in the previous section were also tested on GHOST cells. Eight of them were obtained from infants at acute infection while 16 were from children in the chronic stage. In addition, eight NSI viral isolates from different phases of infection were used as R5 control. As shown in Tables 1 and 3, almost 80\% (19/24) of the SI strains indistinctly used CCR5 or CXCR4 receptos, and there was no different-distribution in the early compared to the late stage of infection: $75 \%$ and $82 \%$, respectively (Table 3 ). Thus, SI variants evaluated on GHOST cell lines behaved predominantly as dual R5X4 tropic (Table 1). Of note, there was a clear discrepancy in coreceptor usage of SI variants between the two cells systems tested, in particular of SI isolates obtained in the early stage of infection.

Out of the remaining five isolates, three (\#371, \#064 and \#584) were classified as $\mathrm{X} 4$. Of interest, the initial primary PBMC co-culture supernatants of the three latter cases carried a mixed R5 and X4 variants when tested on GHOST transfected cells. Surprisingly, the remaining two of the SI isolates (\#526 and \#184) appeared as exclusively R5 variant on GHOST transfected cells, although they were previously selected on infected MT-2 cells that only carry CXCR4 coreceptors. Therefore, the two latter SI variants tested were considered as R5X4 dual-tropic. These observations support the idea that there are R5X4 dual tropic variants that are less efficient in using CXCR4 than CCR5 as coreceptors. Moreover, MT-2 cell assay seems to be more efficient than GHOST-CXCR4 transfected cells for the detection of low levels of SI strains or variants with low affinity for CXCR4 [32,33].

A semi-quantification of SI viral infectivity (relative to HIV- $1_{\mathrm{LAI}}$ I PBMC) on GHOST cells was performed. We observed that dual-tropic viruses were a heterogeneous group with different co-receptors affinity (Tables 1 and 3) that also depends on infection stage. According to the classification suggested by Huang et al. 2007 [10] in the acute stage only one (\# 604) Dual R or R5X4 case and one (\# 075) Dual X or R5X4 case were observed, while the remaining four dual tropic isolates infected both cell lines equally $(\mathrm{R} 5=\mathrm{X} 4)$. On the other hand, in the chronic stages, four of 13 dual isolates showed preference for the CXCR4 coreceptor and were considered as dual X (R5X4).

\section{Co-receptor usage prediction based on Env-V3 loop sequence}

We further evaluated the accuracy of bioinformatic algorithms for viral tropism prediction of $\mathrm{R} 5, \mathrm{X} 4$, and dual $\mathrm{R} 5 \mathrm{X} 4$ variants that were classified using the PBMC assay and were considered as reference for viral tropism (Table 4). Twenty-four SI variants obtained from MT-2 cell supernatants and five NSI viral isolates previously tested on the two cell systems for viral tropism were analyzed and compared. Direct sequences of the V3 loop are illustrated in Table 4. Bioinformatic algorithms used included: the V3-loop net charge, the " $11 / 25$ rule", the PSSM, and the geno2pheno (Table 4).

V3 loop net charge was calculated by subtracting the number of negatively charged amino acids [D and E] from the number of positively charged ones [K and R] [29]. The SI viruses representing the sum of X4 and R5X4 variants had a mean \pm SD net charge of $6.62 \pm 1.28$, significantly higher than $5.2 \pm 0.45$ of NSI variants sequences $(\mathrm{p}=0.019)$. However, comparison of net charges between X4 variants $(6.8 \pm 1.32)$ and R5X4 dual tropic variants $(6.33 \pm 1.5)$ did not differ significantly. Although SI variants had a tendency to increase the net charge along time of infection, they did not differ statistically.

The "11/25 rule" classifies viruses as CXCR4-using when they carry positively charged amino acids, Lysine (K) or Arginine (R) at positions 11 and/or 25 of the V3 loop. As illustrated in Table 4, although that all viral isolates studied were selected as SI variants $(n=24)$, only 16 harbored basic amino acid at position 11 or 25 . At primary infection, only three of the eight SI variants (37\%) carried basic amino acid at position 11 or 25 (\#075, \#507 and \#051), and none at both positions together. Of the 16 SI isolates from late stages of infection, 13 harbored basic amino acid at positions $11 / 25$, including three (\#034, \#037 and \#064) at both of them. The remaining three SI viruses did not carry a basic amino acid at any of the positions 11/25, similar to the five NSI isolates analyzed as controls. These results indicate that the $11 / 25$ rule had low sensitivity for predicting CXCR4 usage on bulk (population-based) sequences from SI isolates from early infection, and is relatively reliable for SI isolates from late stage of infection with a sensitivity of $81 \%$. On the other hand, no differences were observed in the frequency of basic amino acids at position 11/25 between $\mathrm{X} 4$ and R5X4.

Bioinformatics-based genotypic predictors, such as the PSSM (PSSM ${ }_{\mathrm{X} 4-\mathrm{R} 5}$ or PSSM ${ }_{\text {II-NSI }}$ ) and the Geno2pheno (G2P) [30,31], were tested on the same set of SI samples. Out of 24 SI $e n v$ sequences analyzed, 17 and 20 were X4 for PSSM and G2P, respectively (Table 4). These two algorithms were not adequate in predicting the viral tropism for HIV-1 isolates from primary infection ( $50 \%$ for PSSM and of $62 \%$ for G2P, $n=8$ ). In the chronic stage, those algorithms had a concordance with the SI phenotype of $81 \%$ and $93 \%$, respectively. As expected, no differences were observed between the different PSSM matrices, PSSM ${ }_{\mathrm{X} 4-\mathrm{R} 5}$ or PSSM ${ }_{\text {SI-NSI. }}$ Therefore, the results obtained using the 11/25 rule, the PSSM, and theG2P algorithms were similar and relatively efficient in predicting HIV-1 co-receptor usage for SI strains in late stages of infection, but were not adequate to predict viral tropism at early stages. All env sequences from NSI isolates were classified as R5 using the PSSM and the G2P algorithms.

Differences between the genotypic profile of X4 and Dual X4R5 were also evaluated. HIV-1 X4 variants had a significantly higher PSSM score with a median of -1.55 (IQR -5.98 to -0.13 ) than dual-R5X4 variants with a median score of $-6,210$ (IQR -8.325 to -4.415 ) with a $p$ value of 0.0253 . Therefore, the PSSM score may be useful to predict the probability of an SI variant being R5X4 or X4. Conversely, the geno2pheno model could not predict differences between these two SI variants. 
Open Access

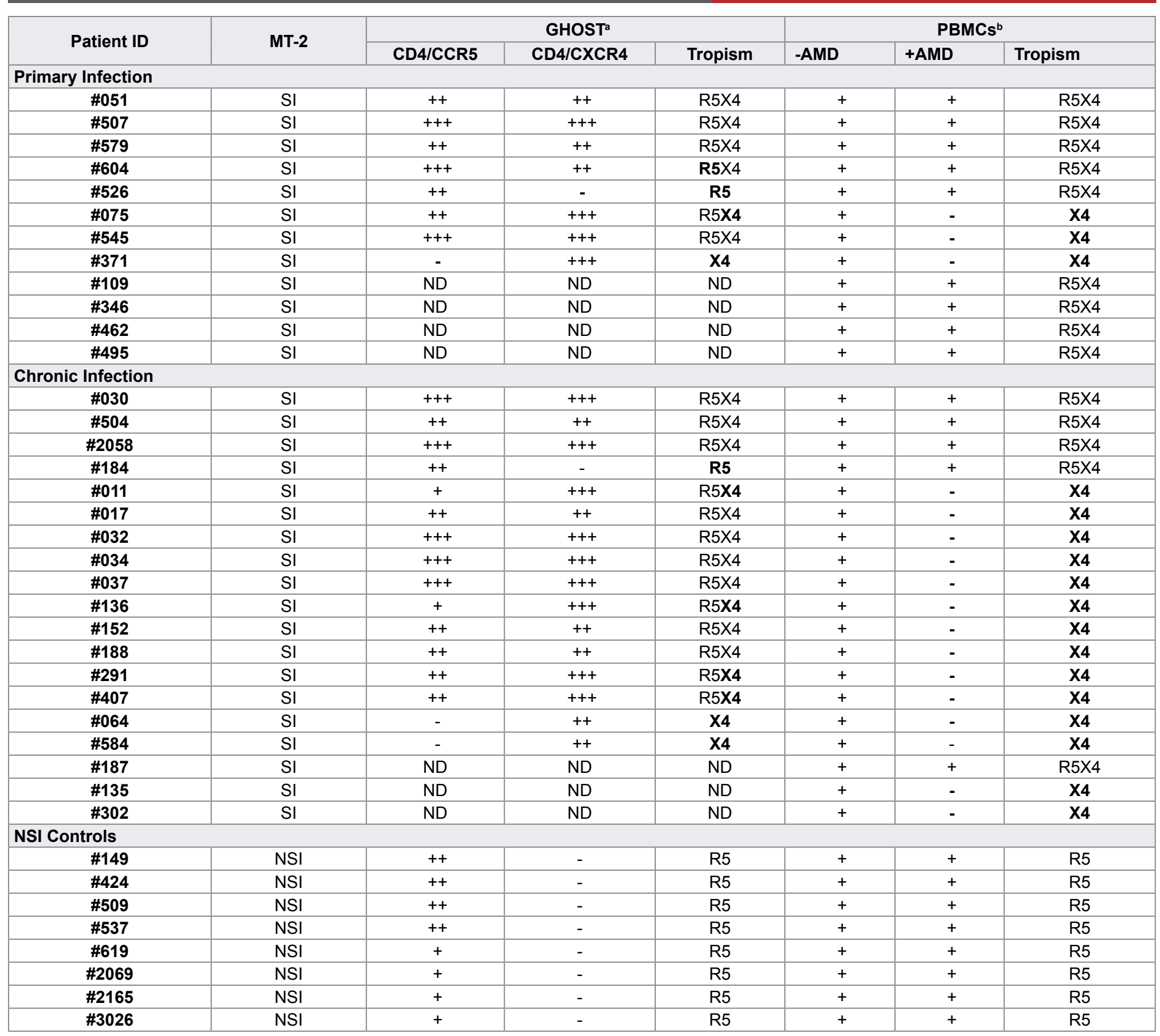

Table 1: Phenotypic determination of coreceptor usage of SI HIV-1 isolates on different cell system.

aGHOST: Levels of infectivity on GHOST-CCR5 and GHOST-CXCR4 cells;

$(+/-)$ occasional, $(+)$ low, $(++)$ moderate and (+++) high according to the classification of Huang et al. 2007 [10].

R5X4: Dual $R(R 5>X 4)$ group included viral isolates with higher infectivity on cells expressing CCR5 compared to CXCR4.

R5X4: Dual $(R 5=X 4)$ group was defined as viral isolates having similar infectivity on both type cell lines, GHOST-CCR5 and GHOST- CXCR4.

R5X4: The Dual X (X4>R5) group included viral isolates with a higher infectivity on cells expressing CXCR4 compared to CCR5.

'PBMCs: (+) p24-Ag positive.

${ }^{*}$ CXCR4-using was confirmed by the inhibition of viral infection with AMD3100 (10ug/ml), a specific CXCR4 inhibitor.

ND: not determined

Table 5 summarizes the sensitivity of the four genotypic algorithms based on the Env V3 loop for SI HIV-1. The net charge, the 11/25 rule, the PSSM and, the Geno2pheno algorithms were not suitable for prediction of coreceptor usage of SI HIV-1 isolates from primary pediatric infection. The highest concordance between cultures and genotypic methods for identification of X4 or Dual Tropic variants was observed in the chronic stage of infection.

To rule out a possible viral selection in cell culture, we further compared
SI HIV-1 populations recovered from different sources. We analyzed V3 loop sequences from viruses present in primary culture supernatants (SN-PBMC) and MT-2 culture supernatants (SN-MT-2) of eight patients with SI HIV-1 strains. In four cases (\#051, \#371, \#504 and \#584) V3 loop sequences were also obtained directly from infected GHOST cell line supernatants. As illustrated in Table 6, all the SI V3 loop sequences from supernatants of MT-2 cells and of primary PBMC cocultures were identical, including three of four cases from transfected GHOST cell line supernatants. The discordant sequence (\#051), which was obtained from 


\begin{tabular}{|c|c|c|c|}
\hline Stage of infection & $\mathbf{n}$ & $\mathbf{R 5 X}$ & $\mathbf{X 4}$ \\
\hline Acute infection & $12(100 \%)$ & $9(75 \%)$ & $3(25 \%)$ \\
\hline Chronic infection & $19(100 \%)$ & $5(26 \%)$ & $14(74 \%)$ \\
\hline
\end{tabular}

Table 2: Coreceptor usage of SI HIV-1 variants tested on peripheral blood mononuclear cells

R5X4: When PBMC post-infection has a supernatant p24+, in the presence or absence of the CXCR4 blocker AMD-3100, the SI variant was considered as dual-tropic.

X4: When PBMC post-infection in the presence of the CXCR4 blocker AMD-3100 has an undetectable of p24 in the supernatant but detectable in the absence of the blocker, the SI variants were considered as an X4 strain.

\begin{tabular}{|c|c|c|c|c|c|c|}
\hline $\begin{array}{c}\text { Stage of } \\
\text { infection }\end{array}$ & Total & R5 & & R5X4 & & X4 \\
\hline $\begin{array}{c}\text { Acute } \\
\text { infection }\end{array}$ & $\mathbf{n}$ & 1 & 1 & 4 & 1 & 1 \\
\hline$(\%)$ & 100 & 12.5 & & $\mathbf{7 5}$ & & 12.5 \\
\hline $\begin{array}{c}\text { Chronic } \\
\text { infection }\end{array}$ & 16 & 1 & - & 9 & 4 & 2 \\
\hline$(\%)$ & 100 & 6 & & $\mathbf{8 2}$ & & 12 \\
\hline
\end{tabular}

Table 3: Classification of SI HIV-1 isolates based on infectivity of CCR5expressing and CXCR4-expressing GHOST cells

R5: When the viral isolates only infected GHOST-CCR5 cells. R5X4: Dual $R(R 5>X 4)$ group includes viral isolates infecting both cell types but with higher infectivity on GHOST-CCR5 cells than GHOST-CXCR4. R5X4: Dual $(R 5=X 4)$ group was defined as viral isolates having similar infectivity on both GHOST-CCR5 and GHOST-CXCR4 cell lines.

R5X4: Dual $X(X 4>R 5)$ group includes viral isolates with a higher infectivity on GHOST cells expressing CXCR4 than on GHOST- CCR5.

X4: SI viral isolates that only infected GHOST-CXCR4 cells.

infected GHOST-CXCR4 cells, showed changes of amino acid residues at positions $7(\mathrm{~N} / \mathrm{T})$ and $13(\mathrm{Q} / \mathrm{R})$, causing an increase of the net charge compared to the other sequences from supernatants PBMC, MT-2, and GHOST-CCR 5 cells from the same patient. With the exception of the last case, no significant amino acidic sequence variation was detected on the V3 loop in the successive passages through different types of cells.

\section{Discussion}

SI HIV-1 variants could be classified as X4 o X4R5 depending on the cellular system employed to evaluate viral tropism. In GHOST cell lines SI HIV-1 were predominantly R5X4 dual-tropic all along time of pediatric infection. However, when evaluated on PBMCs, SI isolates from acute infection were mainly R5X4 dual tropic while in the chronic stage they were mainly $\mathrm{X} 4$ variants. The differences in SI coreceptor usage at different times of infection observed in PBMCs may reflect the evolution of viral tropism from dual-R5X4 to X4, supporting the idea that X4/SI variants may evolve from NSI strains instead of being a minoritary transmitted population.

All methods compared in this study were acceptably solid in determining HIV tropism (with MT-2 infection as the gold standard method) for isolates from chronically infected patients, though the sensitivity of bioinformatic algorithms for tropism prediction of HIV variants from acute infection was really poor (37-62\%). In this study, it was also shown that selection of SI HIV-1 variants on infected MT-2 cells followed by further evaluation of coreceptor usage may distinguish between mixed R5/X4 and dual tropic R5X4 variants, information that cannot be obtained from genotypic computational algorithms.

We observed that out of the SI HIV-1 variants that behaved as R5X4 dual tropic strains in GHOST transfected cell lines, 33\% of those isolated at primary infection and $77 \%$ of those isolated in the chronic stage were exclusively X4 variants when tested on PBMCs. Although PBMC express surface CCR5 molecules, some SI variants that are dual-tropic on GHOST cells seem to be unable to infect PBMCs in vitro in the presence of CXCR4 blockers. In agreement with Yi et al. [20], the inability of dual R5X4 tropic strains to infect CCR5+ lymphocytes raised the possibility to have a less efficient interaction with CCR5 than R5 strains. Contrary to our findings, Toma et al. [11] found a generally concordance in CCR5 coreceptor usage of dual tropic HIV-1 viruses conducted in cell lines and in PBMC assays. The discrepancies between our results and those of Toma et al. [11] may be attributable to differences in the cell system used. However, and as suggested by our results, Toma et al. [11] concluded that dual viruses vary broadly in their ability to use CXCR4 and CCR5 for infection.

Since lymphocytes and macrophages are the primary HIV-1 target cells, it is reasonable to assume that apparent tropism of SI variants in vivo may more closely resembles those obtained in PBMCs than in GHOST cells.

The heterogenety of coreceptor usage among the R5X4 viruses has previously been described by Glushakova et al. [34], and was further confirmed by Huang et al. [33] by molecular analysis of HIV-1 env [33]. However, the differences in coreceptor-dependent infection capacity observed in GHOST cells could not be replicated on PBMCs. According to our study the information obtained on coreceptor usages performed on both cell systems may be complementary since the SI HIV-1 variants that were classified as $\mathbf{R 5}$ or R5X4 by GHOST cells, in the PBMC system behaved as R5X4 while dual tropic variants considered as R5X4 or X4 on GHOST cells, behaved as $\mathbf{X} \mathbf{4}$ on PBMCs. Therefore, it seems that SI variants can suffer evolutionary changes in coreceptor tropism along time of infection from R5 or R5X4 to R5X4 to R5X4 to X4 confirming the concept expressed by Glushakova et al. [34] in 1999 and suggesting that R5X4 viruses represent continuous evolutionary stages ranging from R5 to X4. Although the assay is time consuming, the previous selection of SI variants from infected MT-2 cells demonstrates whether SI supernatants are composed of mixed populations of R5 and X4 variants or are in fact R5X4 dual tropic strains. In the latter variants, evaluation using the GHOST cell system provides information concerning coreceptor usage efficiencies that may be useful for further implementation of antiretroviral coreceptor-blocker therapy.

The current availability of ARV drugs targeting viral coreceptors highlights the need of assays with a rapid and high precision in detecting coreceptor tropism of HIV-1 variants before drug administration. Trofile assay [33] and methods based on env V3 loop gene sequences [29-31] are currently approved by the CDC (US) for the screening of HIV-1 X4 or R5X4 variants. Since MT-2 cell assay tested with viral stocks has a $98 \%$ concordance with the Trofile assay [33], our studies done on MT-2 cells may be considered almost as accurate as the Trofile assay. Viral tropism prediction sensitivity varies broadly depending on the method used for prediction. Though bioinformatic methods based on V3 loop env gene sequences are the simplest and fastest, they had very poor prediction accuracy, ranging between 71 to $91 \%[16,17,35]$. Our studies on the V3 loop of the SI variants using four of the most commonly used bioinformatic algorithms, revealed striking differences in the results between SI variants isolated in the acute and chronic stages of infection. Although an acceptable concordance between phenotypic and genotypic methods using sequences of chronic stages of pediatric infection was observed, the bioinformatic algorithms failed to predict X4 variants isolated from patients with acute infection, suggesting a probable acquisition of mutations improving CXCR4 usage with time of infection. On the other hand, accuracy differed significantly between the four algorithms. G2P had the highest concordance of almost $94 \%$ between SI and CXCR4-usage when variants were from the chronic stage while it fell to $63 \%$ when used on isolates from 


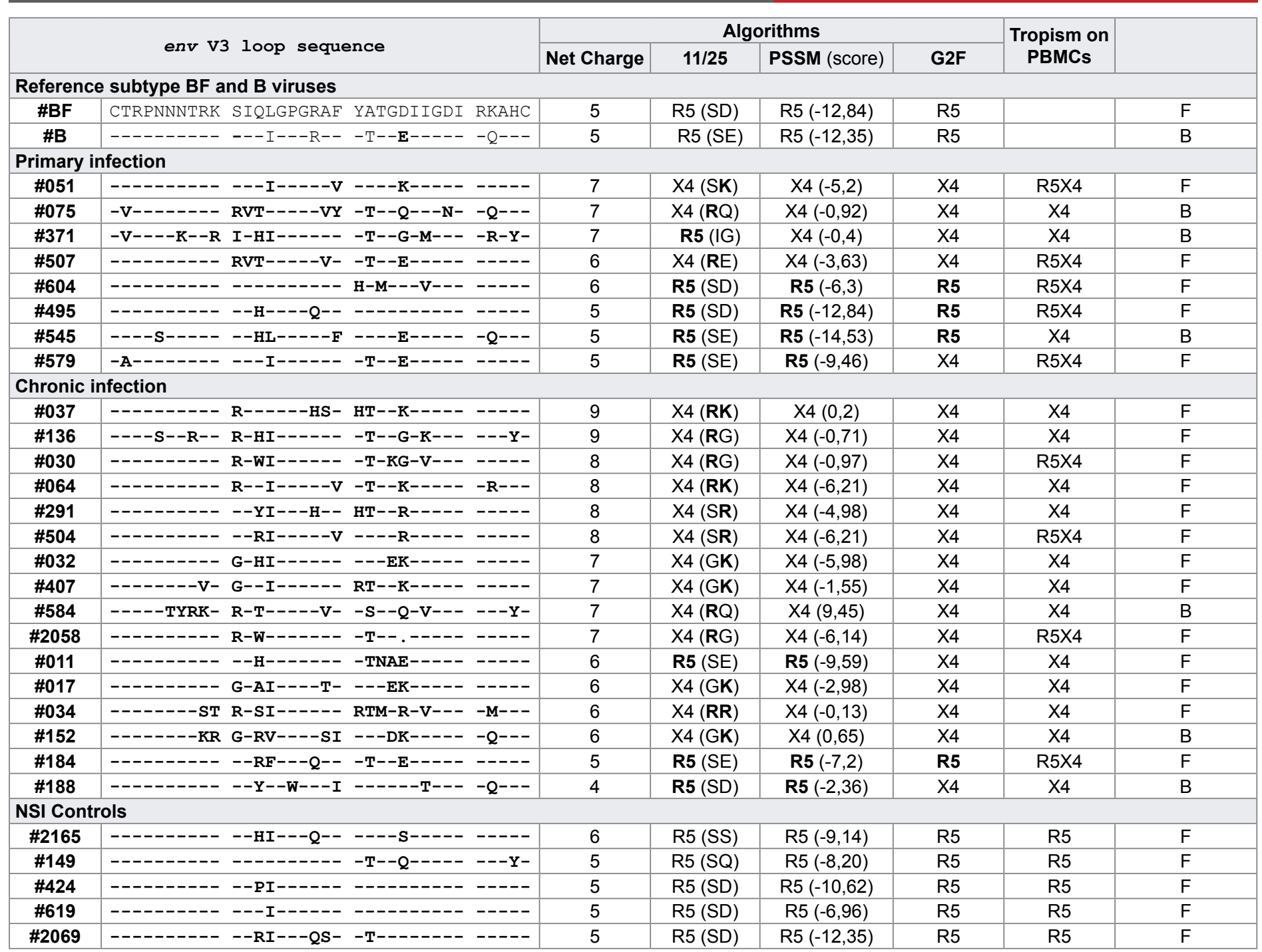

Table 4: Comparison between phenotypic and genotypic determinations of viral tropism of SI HIV-1 isolates

\begin{tabular}{|ll|c|c|c|c|}
\hline Stage of infection & $\mathbf{n}$ & Net Charge (\%) & $\mathbf{1 1 / 2 5}(\%)$ & PSSM (\%) & G2F (\%) \\
\hline Acute infection & 8 & 62,5 & 37 & 60 \\
\hline Chronic infection 16 & 87,5 & 81 & 81 & 94 \\
\hline
\end{tabular}

Table 5: Comparison of sensitivity between four V3 genotype-based algorithms for SI HIV-1 variants obtained at different stages of pediatric infection All SI isolates tested $(n=24)$ were derived from MT-2 cell supernatants.

early primary infection. The PSSM and the $11 / 25$ rule were almost similar with an $81 \%$ of sensitivity for SI variants in the chronic stage of infection lowering to $50 \%$ and $38 \%$ respectively at acute stage. The net charge of SI variants had the lowest sensitivity. Therefore, bioinformatic algorithms for phenotype prediction were not suitable for predicting HIV-1 coreceptor usage in pediatric infection, particularly in acute vertical infection. Since those algorithms were developed based on sequences obtained from isolates of adult-infected individuals, they may have better results in the context of non-vertically-acquired infection, though this should be confirmed. It would also be relevant to establish whether or not HIV-1 X4 and R5X4 dual-tropic strains evolved and change their coreceptor affinity and coreceptor usage capacity among time of infection. If it is the case, there would be implications for CCR5-blocker therapy administration. In addition, as some determinants for CXCR4 usage that reside outside the V3 loop may play an important evolutionary role as HIV-1 variants evolve from R5 to X4-tropic, the inclusion of information of the entire env gene region may probably improve the genotypic coreceptor usage prediction of sequence-based bioinformatic methods $[10,36]$.

Different HIV-1 subtypes can vary in their ability to use CCR5 or CXCR4 coreceptors. Among M viruses, subtype D and CRF01_AE have shown a higher prevalence of CXCR4-using variants when compared to other subtypes, [10,37] though this finding is still controversial [35]. In our population, $\mathrm{BF}$ recombinants represent almost $85 \%$ of the circulating strains [38]. Delgado et al. [39] have recently shown that the correlation between GHOST-cell-culture phenotypic assay and prediction algorithms based on env sequences was very low in a small group of subtype F HIV strains studied. Further studies are needed to assess whether subtypespecific differences in V3 loop sequences may affect HIV tropism.

HIV-1 biological diversity and viral evolution along time of infection 


\begin{tabular}{|c|c|c|c|}
\hline Patient ID & env & e & \\
\hline$\# 037$ SN-PBMC & TRPNNNTRK RIQLGPGHSF & HTTGKIIGDI & RKAHC \\
\hline \#037_SN-MT-2 & --------------------- & ----------- & ----- \\
\hline \#051_SN-PBMC & $---------S--I---R A V$ & YA-------- & ----- \\
\hline$\# 051$ SN-MT-2 & --------- S--I---RAV & YA-------- & ----- \\
\hline \#051 SN-GR5 & --------1 S--I---RAV & YA-------- & ----- \\
\hline \#051_SN-GX4 & $------\mathrm{T}---\mathrm{S}-\mathrm{RI}---\mathrm{RAV}$ & YA-------- & ----- \\
\hline \#075_SN-PBMC & $-\mathrm{V}--------\quad-\mathrm{VT}----\mathrm{RVY}$ & $\mathrm{Y}---\mathrm{Q}---\mathrm{N}-$ & $-Q---$ \\
\hline$\# 075$ SN-MT-2 & $-\mathrm{V}--------\quad-\mathrm{VT}----\mathrm{RVY}$ & $\mathrm{Y}---\mathrm{Q}---\mathrm{N}-$ & $-Q---$ \\
\hline \#371 SN-PBMC & $-\mathrm{V}----\mathrm{K}--\mathrm{R}$ I-HI---RA- & Y---G-M--- & $-R-Y-$ \\
\hline \#371 SN-MT-2 & $-\mathrm{V}----\mathrm{K}--\mathrm{R}$ I-HI---RA- & Y---G-M--- & $-R-Y-$ \\
\hline \#371_SN-GX4 & $-\mathrm{V}----\mathrm{K}--\mathrm{R} \quad \mathrm{I}-\mathrm{HI}---\mathrm{RA}-$ & Y---G-M--- & $-\mathrm{R}-\mathrm{Y}-$ \\
\hline$\# 504 \_S N-P B M C$ & $----------\quad$ S-RI---RAV & $\mathrm{YA}--\mathrm{R}-----$ & ----- \\
\hline \#504 SN-MT-2 & -------- S-RI---RAV & YA--R----- & ----- \\
\hline \#504_SN-GR5 & ---------- S-RI $---\mathrm{RAV}$ & $\mathrm{YA}--\mathrm{R}-----$ & ----- \\
\hline$\# 504$ SN-GX4 & --------- S-RI---RAV & YA--R----- & ----- \\
\hline \#579_SN-PBMC & $-A--------S--I---R A-$ & Y----E----- & ----- \\
\hline \#579_SN-MT-2 & $-\mathrm{A}--------\mathrm{S}--\mathrm{I}---\mathrm{RA}-$ & $Y---E-----$ & ----- \\
\hline$\# 584$ SN-PBMC & ----- TYRK- -- T----RV- & $\mathrm{YS}--\mathrm{Q}-\mathrm{V}---$ & $---Y-$ \\
\hline \#584_SN-MT-2 & ----- TYRK- -- T----RV- & $Y S--Q-V---$ & $---Y-$ \\
\hline \#584_SN-GX4 & ----- TYRK- -- T-----RV- & $\mathrm{YS}--\mathrm{Q}-\mathrm{V}---$ & $---Y-$ \\
\hline
\end{tabular}

Table 6: Comparison of $\mathrm{V} 3$ loop amino acidic sequences of SI HIV-1 variants from primary culture supernatant (SN-PBMC), MT-2 culture supernatant (SN-MT-2), and GHOST culture supernatant (SN-GX4 and SN-GR5)

are two key factors of HIV-1 immunopathogenesis. It is relevant to note that coreceptor usage in vitro may not necessarily predict the viral pathway in vivo, a fact that may lead to the wrong implementation of certain ARV drugs as CCR5 blockers. Differences in SI HIV-1 phenotypic coreceptor usage along time of infection in addition to the differences in the prediction of HIV-1 coreceptor usage according to the method used need further and more thorough research in order to warrant safe and effective implementation of coreceptor blockers as antiretroviral therapy.

\section{Acknowledgments}

The authors are gratefully to Mrs María del Cármen Galvez for the excellent technical assistance. The research was supported by CONICET, through PIP 6057-2004 and ANPCyT through PICT/BID 25830-2004.

\section{References}

1. Tersmette M, de Goede RE, Al BJ, Winkel IN, Gruters RA, et al. (1988) Differential syncytium-inducing capacity of human immunodeficiency virus isolates: frequent detection of syncytium-inducing isolates in patients with acquired immunodeficiency syndrome (AIDS) and AIDSrelated complex. J Virol 62: 2026-2032.

2. Poveda E, Briz V, Quiñones-Mateu M, Soriano V (2006) HIV tropism: diagnostic tools and implications for disease progression and treatment with entry inhibitors. AIDS 20: 1359-1367.

3. Brumme ZL, Goodrich J, Mayer HB, Brumme CJ, Henrick BM, et al. (2005) Molecular and clinical epidemiology of CXCR4-using HIV-1 in a large population of antiretroviral-naive individuals. J Infect Dis 192: 466-474.

4. de Mendoza C, Rodriguez C, García F, Eiros JM, Ruíz L, et al. (2007) Prevalence of $\mathrm{X} 4$ tropic viruses in patients recently infected with HIV-1 and lack of association with transmission of drug resistance. J Antimicrob Chemother 59: 698-704.

5. Crudeli CM, Aulicino PC, Rocco CA, Bologna R, Mangano A, et al. (2012) Relevance of early detection of HIV-1 SI/CXCR4-using viruses in vertically infected children. AIDS Res Hum Retroviruses 28: 685-692.

6. Koot M, Keet IP, Vos AH, de Goede RE, Roos MT, et al. (1993) Prognostic value of HIV-1 syncytium-inducing phenotype for rate of CD4+ cell depletion and progression to AIDS. Ann Intern Med 118: 681-688.
7. Richman D, Bozzette SA (1994) The impact of the syncytium-inducing phenotype of human immunodeficiency virus on disease progression. $J$ Infect Dis 169: 968-974.

8. Kopka J, Batalla M, Mangano A, Mecikovsky D, Bologna R, et al. (2002) Relevance of viral phenotype in the early AIDS outcome of pediatric HIV-1 primary infection. Pediatr Res 52: 475-480.

9. Japour AJ, Fiscus SA, Arduino JM, Mayers DL, Reichelderfer PS, et al. (1994) Standardized microtiter assay for determination of syncytiuminducing phenotypes of clinical human immunodeficiency virus type 1 isolates. J Clin Microbiol 32: 2291-2294.

10. Huang W, Eshleman SH, Toma J, Fransen S, Stawiski E, et al. (2007) Coreceptor tropism in human immunodeficiency virus type 1 subtype $\mathrm{D}$ : high prevalence of CXCR4 tropism and heterogeneous composition of viral populations. J Virol 81: 7885-7893.

11. Toma J, Whitcomb JM, Petropoulos CJ, Huang W (2010) Dual-tropic HIV type 1 isolates vary dramatically in their utilization of CCR5 and CXCR4 coreceptors. AIDS 24: 2181-2186.

12. Douek DC, McFarland RD, Keiser PH, Gage EA, Massey JM, et al. (1998) Changes in thymic function with age and during the treatment of HIV infection. Nature 396: 690-695.

13. Gaulton G, Scobie J, Rosenzweig M (1997) HIV-1 and the thymus. AIDS 11: 403-414.

14. Correa R, Munoz-Fernandez MA (2001) Viral phenotype affects the thymic production of new T cells in HIV-1-infected children. AIDS 15: 1959-1963.

15. Wilkin TJ, Goetz MB, Leduc R, Skowron G, Su Z, et al. (2011) Reanalysis of coreceptor tropism in HIV-1-infected adults using a phenotypic assay with enhanced sensitivity. Clin Infect Dis 52: 925-928.

16. Low AJ, Dong W, Chan D, Sing T, Swanstrom R, et al. (2007) Current V3 genotyping algorithms are inadequate for predicting X4 co-receptor usage in clinical isolates. AIDS 21: 17-24.

17. Garrido C, Roulet V, Chueca N, Poveda E, Aguilera A, et al. (2008) Evaluation of eight different bioinformatics tools to predict viral tropism in different human immunodeficiency virus type 1 subtypes. J Clin Microbiol 46: 887-891.

18. Recordon-Pinson P, Soulié C, Flandre P, Descamps D, Lazrek M, et al. (2010) Evaluation of the genotypic prediction of HIV-1 coreceptor use versus a phenotypic assay and correlation with the virological response to maraviroc: the ANRS GenoTropism study. Antimicrob Agents Chemother 54: 3335-3340.

19. Trabaud MA, Icard V, Scholtes C, Perpoint T, Koffi J, et al. (2012) Discordance in HIV-1 co-receptor use prediction by different genotypic algorithms and phenotype assay: intermediate profile in relation to concordant predictions. J Med Virol 84: 402-413.

20. Yi Y, Shaheen F, Collman RG (2005) Preferential use of CXCR4 by R5X4 human immunodeficiency virus type 1 isolates for infection of primary lymphocytes. J Virol 79: 1480-1486.

21. Goodenow MM, Collman RG (2006) HIV-1 coreceptor preference is distinct from target cell tropism: a dual-parameter nomenclature to define viral phenotypes. J Leukoc Biol 80: 965-972.

22. Hollinger FB, Bremer JW, Myers LE, Gold JW, Mc Quay L (1992) Standardization of sensitive human immunodeficiency virus coculture procedures and establishment of a multicenter quality assurance program for the AIDS Clinical Trials Group. J Clin Microbiol 30: 1787-1794.

23. Albert J, Fenyo EM (1990) Simple, sensitive, and specific detection of human immunodeficiency virus type 1 in clinical specimens by polymerase chain reaction with nested primers. J Clin Microbiol 28: 1560-1564.

24. Moore JP, Kitchen SG, Pugach P, Zack JA (2004) The CCR5 and CXCR4 coreceptors--central to understanding the transmission and pathogenesis of human immunodeficiency virus type 1 infection. AIDS Res Hum Retroviruses 20: 111-126. 
25. Vödrös D, Fenyö EM (2001) Quantitative evaluation of HIV and SIV coreceptor use with GHOST(3) cell assay. Methods Mol Biol 304: 333-342.

26. Delwart EL, Herring B, Rodrigo AG, Mullins JI (1995) Genetic subtyping of human immunodeficiency virus using a heteroduplex mobility assay. PCR Methods Appl 4: S202-S216.

27. Thompson JD, Gibson TJ, Plewniak F, Jeanmougin F, Higgins DG (1997) The CLUSTAL_X windows interface: flexible strategies for multiple sequence alignment aided by quality analysis tools. Nucleic Acids Res 25: 4876-4882.

28. Hall TA (1999) BioEdit: a user-friendly biological sequence alignment editor and analysis program for Windows 95/98/NT. Nucleic Acids Symp Ser 41: 95-98.

29. Fouchier RA, Groenink M, Kootstra NA, Tersmette M, Huisman HG, et al. (1992) Phenotype-associated sequence variation in the third variable domain of the human immunodeficiency virus type $1 \mathrm{gp} 120$ molecule. J Virol 66: 3183-3187.

30. Jensen MA, Li FS, van 't Wout AB, Nickle DC, Shriner D, et al. (2003) Improved co-receptor usage prediction and genotypic monitoring of R5 to X4 transition by motif analysis of HIV type 1 env V3 loop sequences. J Virol 77: 13376-13388.

31. Lengauer T, Sander O, Sierra S, Thielen A, Kaiser R (2007) Bioinformatics prediction of HIV coreceptor usage. Nat Biotechnol 25: 1407-1410.

32. Lee B, Sharron M, Montaner LJ, Weissman D, Doms RW (1999) Quantification of CD4, CCR5, and CXCR4 levels on lymphocyte subsets, dendritic cells, and differentially conditioned monocytederived macrophages. PNAS 96: 5215-5220.
33. Coakley E, Reeves JD, Huang W, Mangas-Ruiz M, Maurer I, et al. (2009) Comparison of human immunodeficiency virus type 1 tropism profiles in clinical samples by the Trofile and MT-2 assays. Antimicrob Agents Chemother 53: 4686-4693.

34. Glushakova S, Yi Y, Grivel JC, Singh A, Schols D, et al. (1999) Preferential coreceptor utilization and cytopathicity by dual-tropic HIV1 in human lymphoid tissue ex vivo. J Clin Invest 104: R7-R11.

35. Huang W, Toma J, Fransen S, Stawiski E, Reeves JD, et al. (2008) Coreceptor tropism can be influenced by amino acid substitutions in the gp41 transmembrane subunit of human immunodeficiency virus type 1 envelope protein. J Virol 82: 5584-5593.

36. Pastore C, Nedellec R, Ramos A, Pontow S, Ratner L, et al. (2006) Human immunodeficiency virus type 1 coreceptor switching:V1/V2 gain-of-fitness mutations compensate for $\mathrm{V} 3$ loss-of-fitness mutations. J Virol 80: 750-758.

37. Frange P, Chaix ML, Raymond S, Galimand J, Deveau C, et al. (2010) Low frequency of CXCR4-using viruses in patients at the time of primary non-subtype-B HIV-1 infection. J Clin Microbiol 48: 3487-3491.

38. Aulicino PC, Bello G, Guimaraes ML, Ruchansky D, Rocco C, et al. (2011) Longitudinal analysis of HIV-1 BF1 recombinant strains in vertically infected children from Argentina reveals a decrease in CRF12 BF pol gene mosaic patterns and high diversity of BF unique recombinant forms. Infect Genet Evol 11: 349-357.

39. Delgado E, Fernández-García A, Vega Y, Cuevas T, Pinilla M, et al. (2012) Evaluation of genotypic tropism prediction tests compared with in vitro co-receptor usage in HIV-1 primary isolates of diverse subtypes. J Antimicrob Chemother 67: 25-31. 